\title{
True dedication to clinical research: the Clinical Center of the National Institutes of Health receives the 2011 Mary Woodard Lasker Award for Public Service
}

\begin{abstract}
The
Holy Grail for the majority of physician-scientists is for their work to translate into something beneficial for human health. As the largest hospital in the United States devoted entirely to clinical research, the Clinical Center of the NIH (Figure 1) is the place where many have reached this goal. The Albert and Mary Lasker Foundation is honoring the Clinical Center of the NIH with the 2011 Mary Woodard Lasker Award for Public Service for its many years of service as a model research hospital that provides highquality patient care; enables rapid translation of laboratory observations into new, innovative approaches for diagnosing, treating, and preventing disease; and trains thousands of physician-scientists who lead the way in translational biomedical research.
\end{abstract}

\section{America's research hospital}

Construction of the Clinical Center of the NIH was started in November 1948, and the facility formally opened in 1953. The vision that Congress had for the Clinical Center was outlined by Rep. Frank B. Keefe, chair of the House Appropriations subcommittee that approved federal funding for the facility, when he said, "[This Committee] . . . is supremely interested in this matter of providing proper clinical research facilities that will bring the sufferer, the human patient, into direct contact with the researchers" (1). These ideas were retained by successive Congressional oversight committees and are reflected in the current vision of the Clinical Center, which is to "... lead the global effort in training today's investigators and discovering tomorrow's cures." Efforts over the years to realize these goals have led to the Clinical Center becoming the largest hospital in the country dedicated entirely to clinical research - currently, in a given year there are 6,000 inpatient admissions and 95,000 outpatient visits. As such, it is often referred to as America's research hospital.

\section{Facilitating clinical research throughout the $\mathrm{NIH}$}

The function of the Clinical Center is to provide a setting for researchers at the other NIH Institutes and Centers to perform clinical research. Currently, 17 of the other Institutes and Centers have projects running at the Clinical Center. As it was

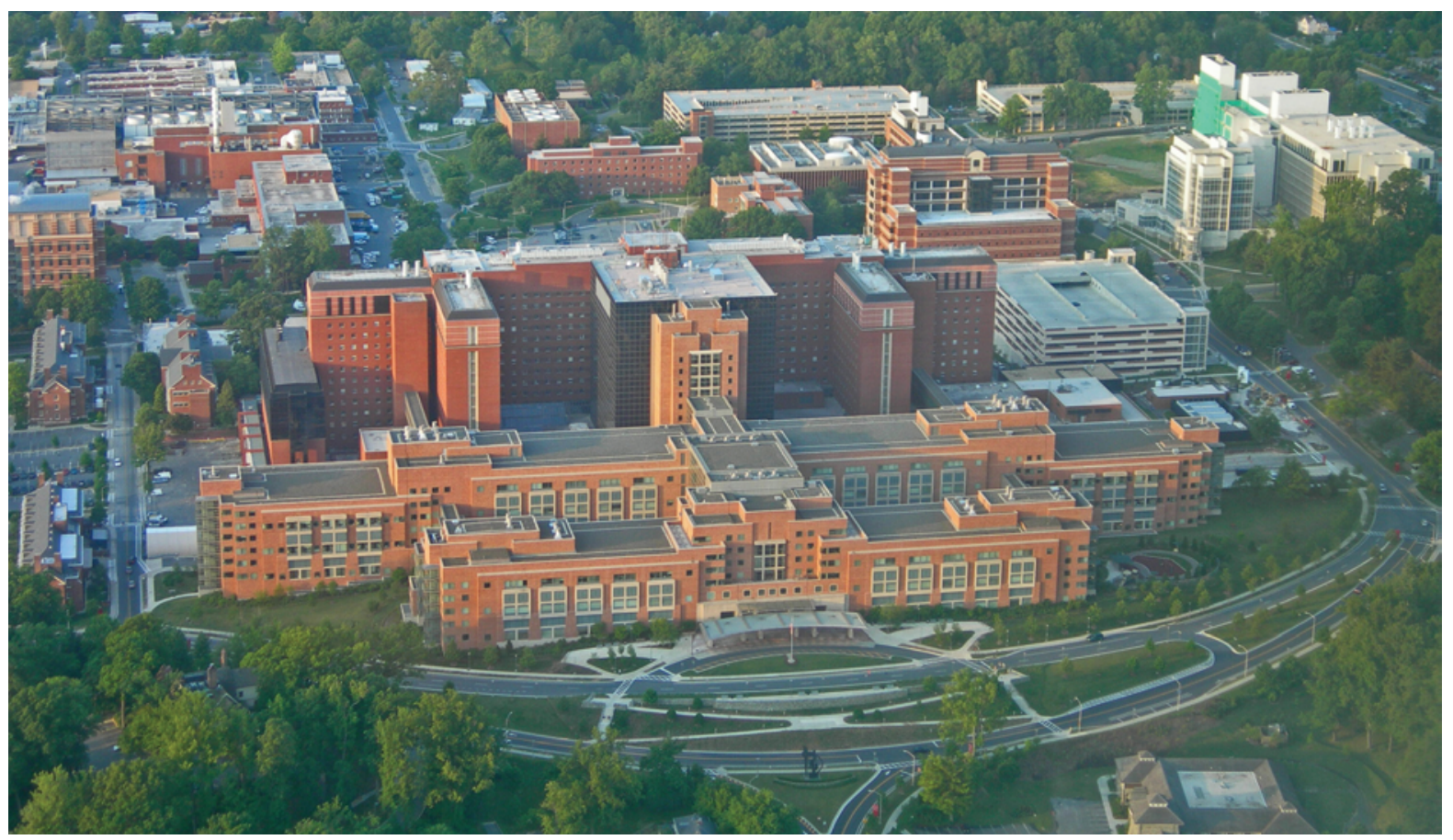

Figure 1

The Mark O. Hatfield Clinical Research Center, the newest addition to the Clinical Center of the NIH, which is being honored with the $2011 \mathrm{Mary}$ Woodard Lasker Award for Public Service for providing innovative therapy and high-quality patient care, treating rare and severe diseases, and producing outstanding physician-scientists since its inception. Aerial photograph, taken in May 2007, courtesy of the NIH Clinical Center. 


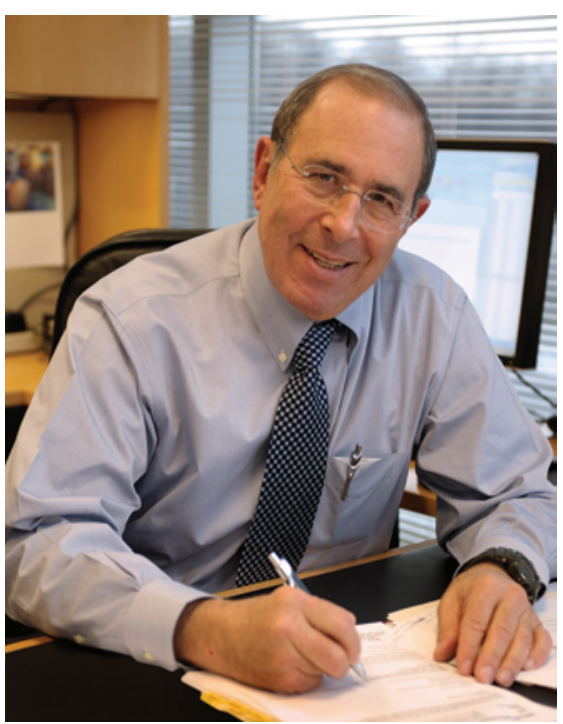

Figure 2

John I. Gallin, Director of the Clinical Center of the NIH since 1994. Photograph courtesy of the NIH Clinical Center.

when the Clinical Center first opened, the National Cancer Institute (NCI) is the biggest user of the Clinical Center - in June 1953, the NCI used 35 of 150 beds, and it currently runs almost one-third of ongoing projects. Although the main job of Clinical Center staff is to facilitate the projects run by researchers at the other NIH Institutes and Centers, they also do their own research, and Clinical Center employees run $6 \%$ of current projects.

The staff of the Clinical Center provides core services such as anesthesiology, rehabilitation, and radiology; the surgeons and physicians are employed by the Institutes and Centers themselves. The Clinical Center also has a state-of-the-art pharmacy that has a good manufacturing practices (GMP) facility in which customized investigational drug formulations can be manufactured for the many first-in-human clinical trials conducted at the Center; among the projects currently underway at the Clinical Center, $45 \%$ are clinical trials, and of these, $90 \%$ are first-in-human phase I or II studies; the remaining $55 \%$ of projects are natural history studies of disease pathogenesis. Another resource available to researchers using the Clinical Center is its GMP cell processing facility, which can generate large numbers of bone marrow-derived and mesenchymal stem cells for clinical use. According to John I. Gallin (Figure 2), the current Director of the Clinical Center, who spoke with the JCI recently, the facility also has the potential to generate embryonic stem cells and induced pluripotent stem cells for clinical studies, if federal go-ahead for such projects is granted.

\section{Patients as partners}

In his address to the collected dignitaries upon laying the cornerstone of the Clinical Center on June 22, 1951, President Harry S. Truman said, "Medical care is for the people and not just for the doctors - and the rich. Our objective must be to make the best modern medical care available to more and more people" (2). Gallin echoed Truman's comments when he spoke with the JCI, saying that in his view, what the Clinical Center is all about is "providing hope and health to people with disease and their families, to everyone."

On July 6, 1953, Charles Meredith, a Maryland farmer with prostate cancer, was the first patient admitted to the Clinical Center (Figure 3). Today, patients come to the Clinical Center not just from all parts of America, but from all around the world. Importantly, the researchers facilitate the travel of many of the patients to the Clinical Center. This extensive geographical reach, with resources to help patients trav$\mathrm{el}$, is a key factor in the success of the Clinical Center according to Gallin. It means that researchers can form large cohorts of patients, even if the disease they are studying is a rare condition. Currently, researchers working at the Clinical Center are studying almost 400 different rare diseases, which are usually defined as those with a prevalence of fewer than 200,000 affected individuals in the United States.

Only patients with a precise kind or stage of illness under investigation by an NIH researcher are admitted to the Clinical Center for treatment. Once there, however, they receive state-of-the-art care at no cost. Family members also have access to a tremendous number of essential resources. The Edmond J. Safra Family Lodge provides the families of adult patients with a homelike place to stay, while the Children's Inn is a home away from home where sick children and their families can stay together during treatment. The Clinical Center also has a school with grades kindergarten through high school to assist children who need to stay for prolonged periods.

It is the partnership with the patients that makes the Clinical Center an amazing place to work, Harvey Alter, Chief of Clinical Studies and Associate Director of Research in the Department of Transfusion
Medicine at the Clinical Center, told the JCI recently. Alter joined the Department of Transfusion Medicine in 1969 and has been there ever since. Among the many things Alter's research has led to is the cleansing of the American blood supply of hepatitis viruses - work that was honored with the 2000 Albert Lasker Clinical Medical Research Award. However, Alter's passion is his patients, some of whom he has been following for 30 years. He told the JCI that the atmosphere at the Clinical Center is unlike that anywhere else because it enables researchers to build unique doctor/patient relationships, practicing old-time medicine with new technologies. Alter said, "The ethos at the Clinical Center makes it possible to study patients as long as you need to; you don't have to rush them out of the door because they are not a source of income, as they are at other hospitals around the country." This, for him, makes the patients partners in his research, and he and his team recently organized a 30th "birthday" party for a patient they have followed for 30 years - from the point he became infected with a hepatitis virus as a result of a blood transfusion until the present day. Alter says he has learned so much from this patient, and he continues to do so as stored samples taken over the years are continually being tested with new techniques as they are developed, adding, "How can I consider him anything other than a research partner?"

\section{Training the nation's clinical researchers}

At the Clinical Center, there are currently clinical research training initiatives for nearly every level of career development, from a summer internship program for high school students to a sabbatical program for experienced researchers. This plethora of programs is key to the Clinical Center's mission to train the current and next generations of clinical researchers. Training has been a core aspect of the Clinical Center since it opened, and with $8 \%$ of current deans of medicine in the United States having been trained at the Clinical Center, it is clear that the programs have been a huge success.

The NIH Associate Training Program began in 1953 with only 15 fellows. However, word soon spread that it provided physicians interested in clinical research with a way to avoid the draft: service in the uniformed Commissioned Corps of the Public Health Service (PHS) provided a means for physicians to fulfill their military obliga- 


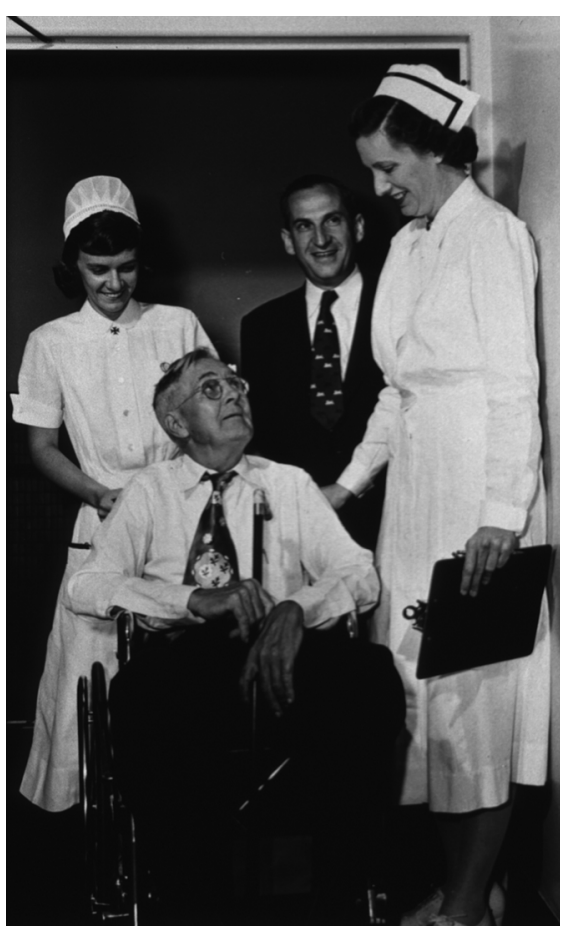

tions, and one option for serving in the PHS was to be assigned to the Associate Training Program. The number of associates admitted to the program swelled during the Vietnam War, peaking at 229 in 1973, and the program participants were known affectionately as the "yellow berets." Many of the physicians who entered the NIH Associate Training Program during the war came with little training in research but got hooked, according to Gallin, on the fluidity between the hospital bed and the lab, and stayed at the NIH, going on to hold key positions. These physicians include Gallin himself and Anthony Fauci (Director of the NIAID).

Other trainees went elsewhere to establish clinical research and/or basic research programs heavily influenced by their time at the Clinical Center. For example, Michael S. Brown and Joseph L. Goldstein - winners of the 1985 Albert Lasker Basic Medical Research Award and Nobel Prize in Physiology or Medicine for their discovery of the basic mechanisms controlling cholesterol metabolism, which ultimately led to the development of cholesterol-lowering statins - were participants in the Associate Training Program between 1968 and 1970. Among the Clinical Center patients they studied during their training were a brother-sister pair with excessively high levels of LDL in their blood, severe atherosclerosis, and a propensity for heart attacks at a young age (a condition later named homozygous

\section{Figure 3}

On July 6, 1953, Charles Meredith (seated), a 67-year-old farmer with prostate cancer, was admitted as the Clinical Center's first patient. He was placed on a hormone therapy protocol by his physician, Roy Hertz (second from right). Also on hand were (left to right) Clinical Center nurses Nadine Luxmore and Elizabeth Walker. Photograph courtesy of the NIH Clinical Center.

familial hypercholesterolemia). Upon leaving the NIH, Brown and Goldstein established a research program at the University of Texas Southwestern Medical School aimed at understanding how the body normally controls the level of cholesterol in the blood and why the level was so high in the children they had seen at the Clinical Center. Brown has said, "If we hadn't seen those children at NIH, we would have never known about this illness, and we would have never worked on the problem" (3).

Today, the Clinical Center has a curriculum designed by Gallin of three courses in clinical research. Last year, using long-distance learning techniques, these courses were taught to 3,000 students in 16 countries and on 5 continents. Gallin and other faculty members have also taken the Clinical Center courses to developing countries, teaching a five-day version of the course "Introduction to the Principles and Practice of Clinical Research" in person at several locations in China and at the University of Ibadan, Nigeria (Figure 4). Gallin says that expanding the Clinical Center training programs beyond the confines of the NIH is a big step and a key part of his vision to make it a resource for those interested in clinical research throughout the world.

\section{Innovative research: past endeavors from bench to bedside and back again}

Over the years, the research facilitated by the Clinical Center has broken new ground in almost every field of biomedical research, and many of those conducting work at the Clinical Center have been recognized for their innovation with Lasker and Nobel awards. For example, Roy Hertz, the oncologist who admitted the first Clinical Center patient, and his colleague Min Chiu $\mathrm{Li}$, were among the recipients of the 1972 Albert Lasker Clinical Medical Research Award for their contribution to the development of chemotherapeutic treatments for malignancies. Hertz and Li were looking for ways to treat women with gestational choriocarcinoma, a highly aggressive cancer of the placenta. They eventually developed a chemotherapeutic cure - the first such cure for a metastatic malignant solid tumor - using a folic acid antagonist that is now known as methotrexate.

Two other recipients of the 1972 Albert Lasker Clinical Medical Research Award worked just down the corridor from Hertz

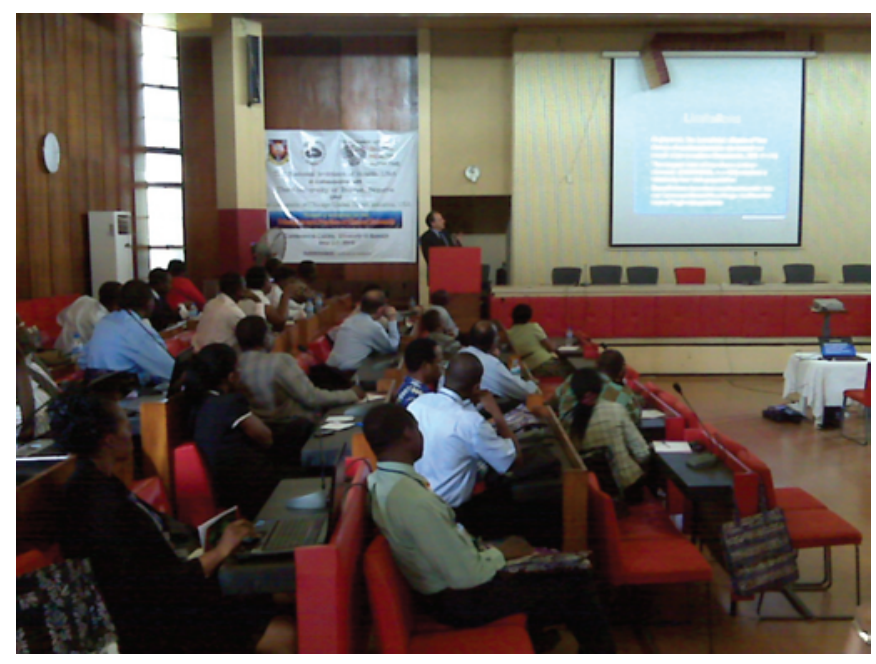

Figure 4

Charles Natanson, head of the anesthesia section of the Clinical Center's Critical Care Medicine Department, teaching part of the Clinical Center's "Introduction to the Principles and Practices of Clinical Research" course at the University of Ibadan, Nigeria, in May 2010. This was the first time an adaptation of this course had been taught in sub-Saharan Africa. Photograph courtesy of the NIH Clinical Center. 
and Li. Emil (Tom) Frei and Emil (Jay) Freireich were developing chemotherapeutic treatments for children with acute lymphocytic leukemia. When they started their work, a diagnosis of leukemia was a death sentence, but after trying many combinations of drugs, Frei and Freireich came upon a combination of four (vincristine, methotrexate, mercaptopurine, and prednisone) that induced a $90 \%$ complete remission rate. Later work by Frei and Freireich as well as others has led to an overall survival rate in children with acute lymphocytic leukemia treated with chemotherapy of almost $80 \%$.

Roscoe Brady and Elizabeth Neufeld were awarded the 1982 Albert Lasker Clinical Medical Research Award for their work on genetic diseases, much of which they conducted at the Clinical Center in the 1960s. Brady and colleagues identified the enzyme defects responsible for the lysosomal storage diseases Gaucher disease, NiemannPick disease, Fabry disease, and Tay-Sachs disease, while Neufeld and colleagues identified the enzyme defects responsible for several of the lysosomal storage diseases known collectively as mucopolysaccharide storage disorders. The work of Brady and Neufeld provided the foundation for the development of assays to diagnose patients with these conditions; strategies to perform prenatal screening for these diseases; and, in the case of Gaucher disease, an effective enzyme replacement therapy.

The Clinical Center also played a key role during the HIV/AIDS public health crisis, awareness of which began emerging there in 1981 - the Center's first patient with AIDS was admitted on June 16, 1981, by NCI investigator Thomas Waldmann, who had no idea why the patient was experiencing multiple infections and a dangerously low white blood cell count. In 1984, Robert Gallo and colleagues at the NCI identified a retrovirus as the cause of AIDS. Investigators in Paris and California simultaneously made the same discovery, and Gallo shared with the leaders of the other groups the 1986 Albert Lasker Clinical Medical Research Award. Further research at the Clinical Center by Samuel Broder and colleagues at the NCI led to the March 1987 FDA approval of azidothymidine (AZT) as a treatment for AIDS.

The Undiagnosed Diseases Program For most people, getting sick results in a relatively quick diagnosis. However, there is a substantial population of individuals with medical conditions that have defied diagnosis by the medical community. The Clinical Center had always been a haven for such individuals, but only those brought in by researchers investigating conditions characterized by a specific set of symptoms. This changed in 2008, when William Gahl, Clinical Director of the National Human Genome Research Institute, began the Undiagnosed Diseases Program. The two key ideas behind the program are to provide answers to patients with mysterious conditions that have long eluded diagnosis and to advance medical knowledge about rare and common diseases.

Thus far, more than 350 patients have come to the Clinical Center as part of the Undiagnosed Diseases Program. However, this is just a small percentage of the total number of cases referred to the program, 4,000. A multidisciplinary team, headed by Gahl, meets every two weeks to discuss in exquisite detail all the patients recently referred to the program. Any patient with a longstanding medical condition that has eluded diagnosis by a referring physician can be considered to have an undiagnosed disease and is of possible interest to the program.

Earlier this year, the first disease unraveled through the Undiagnosed Diseases Program was reported in the New England Journal of Medicine (4). Members of two families with an adult-onset condition associated with progressive and painful arterial calcification in arteries below the waist and in the joints of the hands and feet were admitted to the Clinical Center. One of the families was consanguineous, with five siblings affected by the disease that spared their parents. This led to the hypothesis that the condition could be inherited in an autosomal recessive manner and to SNP analysis to identify regions of the genome for which all five affected siblings were homozygous and both parents heterozygous. Only one such region emerged from the SNP analysis. Genome sequencing of three genes in the region revealed a homozygous nonsense mutation in exon 3 of the $5^{\prime}$-nucleotidase, ecto (NT5E) gene, which encodes CD73, as the cause of disease in the five affected siblings in this family. Nonfunctional mutations in NT5E were also found in the affected individuals of the second family and of an affected individual in Europe.
Gallin told the JCI that the success of the Undiagnosed Disease Program highlights for him one of the key reasons that the Clinical Center exists, which is "to provide hope to patients, especially those with undiagnosed diseases and those with diseases challenging to treat." He hopes that the program will provide a model that other institutions around the country will emulate, such that a series of regional programs that interact with each other might emerge.

Moving forward, another need that has been exposed by the advent of the Undiagnosed Diseases Program is long-term patient care. As Gallin told the JCI, the Clinical Center cannot accommodate all the patients chronically, and the Undiagnosed Diseases Program is actively looking for partners to address this need.

\section{Changes afoot}

The Clinical Center has always been intended to be a global resource for patients and, through the publication of intramural findings, clinical researchers. However, because of its focus on facilitating only intramural research, its reach has been somewhat limited. Following thorough assessment of the Clinical Center in 2010, it was recommended that the Clinical Center expand its scope to become more of a resource for external clinical research communities. While some programs involving collaborations with extramural investigators have been active for a few years, Gallin told the JCI that he sees this as the biggest change that the Clinical Center will undergo in the coming years. However, he is adamant that such changes will not diminish, and will in fact enhance, the position of the Clinical Center as a "monument to clinical research and a beacon to provide hope to patients."

\section{Karen Honey}

1. Mandel R. Beacon of hope, 40: 1953-1993 - the Clinical Center through forty years of growth and change in biomedicine. Washington, D.C., USA: Office of NIH History. http://history.nih.gov/ exhibits/beacon/founding_yea1944-1953Intro. html. Accessed August 17, 2011.

2. Public papers of the presidents, Harry S. Truman 1945-1953: address at the dedication of the National Institutes of Health Clinical Center. Harry S. Truman Library and Museum web site. http://www.trumanlibrary.org/publicpapers/index. php?pid=3498st=8st1. Accessed August 17, 2011.

3. NIH. On the 50th anniversary of the NIH Clinical Center's opening. NIH Clinical Center web site. http://clinicalcenter.nih.gov/about/news/anniver50/ opening.shtml. Accessed August 17, 2011.

4. St. Hilaire C, et al. NT5E mutations and arterial calcifications. NEngl J Med.2011;364(5):432-442. 